\title{
The show must go on. The impacts of SARS-CoV-2 pandemic on cutaneous allergology and patch testing
}

\author{
${ }^{1}$ Department of Dermatology, Hospital de Santa Maria, Centro Hospitalar Universitário Lisboa Norte EPE, Lisbon, Portugal \\ ${ }^{2}$ Universitary Clinic of Dermatology, School of Medicine, University of Lisbon, Lisbon, Portugal \\ ${ }^{3}$ Research Unit of Dermatology, iMM João Lobo Antunes, University of Lisbon, Lisbon, Portugal
}

\section{KEY WORDS \\ Patch test; SARS-CoV-2; drug eruptions; allergic contact dermatitis.}

\section{Corresponding author \\ Miguel Alpalhão \\ Dermatology Department \\ Hospital de Santa Maria \\ Centro Hospitalar Universitário \\ Lisboa Norte EPE \\ 1649-035 Lisbon, Portugal \\ E-mail: migueldbalpalhao@campus.ul.pt}

Doi

10.23822/EurAnnACI.1764-1489.165

\section{Dear Editor,}

While public health campaigns against SARS-CoV-2 pandemic are underway to promote frequent hand washing as one of the ancillary strategies to reduce transmission of the newly identified virus, we are seeing a rise in the frequency of hand dermatitis, many of which are predominantly irritative in nature. While irritative contact dermatitis due to the use of Personal Protection Equipment (PPE) and frequent hand sanitizing has been under the mediatic spotlight we must not forget the significant impact social isolation measures may have on our allergic contact dermatitis patients, who may find themselves deprived of the diagnostic work-up or clinical follow-up necessary for their improvement, during this crisis.

It is well established that allergic contact dermatitis carries a significant impact on quality of life (1). It is also known that the diagnosis of this condition is often delayed and that patch tests are fundamental for allergen identification and avoidance, in many cases (2). This may be particularly important in occupational contact dermatitis in healthcare professionals, where early identification of the culprit allergen may reduce the risk of sick leave, bacterial infection due to skin barrier dysfunction and inadequate use of PPE.

The ongoing SARS-CoV-2 outbreak has impacted clinical practice worldwide. Due to contingency measures, most outpatient appointments and diagnostic procedures are being postponed to reduce patient exposure to high risk environments. The current contingency plan has made patch testing difficult to carry in hospital setting, as two to three visits to a hospital in a week's time carries a significant risk for viral exposure. However, denying patch testing to our patients also takes a toll on their health and quality of life.

Not only will these patients lack the differentiated opinion of an expert in cutaneous allergology, which could provide them 
with the answers and recommendations they need to control their dermatosis, they will also tend to aggravate during this period where frequent washing and disinfection with alcohol based antiseptic solutions may constitute a further aggression to their already sensitized skin. This will inevitably contribute to the perpetuation of the skin barrier disruption-allergen penetration-inflammatory response cycle that characterizes allergic contact dermatitis. This worsening may be disproportionately higher in certain occupations where frequent hand washing is mandatory, as is the case of healthcare professionals.

Furthermore, patch testing is important in the study of patients with severe forms of cutaneous drug eruptions, such as Drug Rash with Eosinophilia and Systemic Symptoms (DRESS), Acute Generalized Exanthematous Pustulosis and even in Stevens-Johnson and Lyell syndromes. Patch testing is also crucial when the suspected culprit drug may be fundamental for current treatment of comorbid diseases (e.g. Anticonvulsants) or when future implications for drug choices for common conditions are significant (e.g. Antibiotics). While the overall sensitivity of patch testing may not be high, it remains one of the most informative tests to date for culprit drug identification, as lymphocytic transformation tests aren't easily accessible and show equally limited sensitivity in most instances, and oral challenge proves carry significant risks when the original dermatosis was severe $(3,4)$. The current constrains may compromise culprit drug identification, which may pose significant hindrances in those instances where patients are taking several different drugs on a daily basis. Paradoxically, these patients with several comorbidities where patch testing may impact clinical management the most tend to be the most vulnerable to severe COVID-19, which would recommend against hospital visits during a pandemic crisis.

Despite all difficulties, it is our duty to provide support to our patients during these challenging times. In the largest tertiary teaching hospital in Portugal, one of the most severely afflicted countries by this outbreak, we have adopted some strategies to help our patients.

All patients referred to our Cutaneous Allergology appointments are being assessed through teleconsultation. At present our main goals are the identification of those patients which need immediate presential assessment and providing all patients with both oral and written recommendations on how to reduce skin aggressions while keeping sanitary measures.

Those cases where patch testing is paramount (about $5 \%$ of all scheduled patients for testing in our center), as is the case of severe drug eruptions, are still being assessed and tested. We try to conduct these appointments in physical spaces separate from those dedicated to COVID-19 patients. Ready-to-use test panels or preparing Finn chambers in advance to patient visit may reduce the duration of initial patch test appointment and contribute towards lower potential viral exposure for patients. We are also reducing the number of hospital visits for patients undergoing patch testing, as patients are being advised to remove the panels at D2 and take appropriate pictures at this timepoint, which are then assessed by the dermatologist at the D4 reading. With these simple measures we can make sure that essential care is being provided to those who need it the most while improving the quality of life of patients who can be assessed when normal clinical activity resumes. Patch testing can be postponed safely in most instances, but it must not be cancelled altogether during this pandemic crisis, as it may provide information with profound implications on the clinical management of some patients.

\section{Conflict of interests}

The authors declare that they have no conflict of interests.

\section{References}

1. Kadyk DL, McCarter K, Achen F, Belsito DV. Quality of life in patients with allergiccontact dermatitis. J Am Acad Dermatol 2003;49(6):1037-48.

2. Nelson JL, Mowad CM. Allergic Contact Dermatitis Patch Testing Beyond the TRUE Test. J Clin Aesthet Dermatol 2010;3(10):4.

3. Romano A, Viola M, Gaeta F, Rumi G, Maggioletti M. Patch Testing in Non-Immediate Drug Eruptions. Allergy Asthma Clin Immunol 2008;4(2):66.

4. Mahajan V, Handa S. Patch testing in cutaneous adverse drug reactions: Methodology, interpretation, and clinical relevance. Indian J Dermatol Venereol Leprol 2013;79(6):836-41. 